\title{
A novel nanoscopic tool by combining AFM with STED microscopy
}

\author{
Benjamin Harke ${ }^{1 * \dagger}$, Jenu Varghese Chacko ${ }^{1,3+}$, Heiko Haschke ${ }^{2}$, Claudio Canale ${ }^{1}$ and Alberto Diaspro ${ }^{1,3^{*}}$
}

\begin{abstract}
We present a new instrument for nanoscopic investigations by coupling an atomic force microscope (AFM) with a super resolution stimulated emission depletion (STED) microscope. This nanoscopic tool allows high resolution fluorescence imaging, topographical imaging and nano-mechanical imaging, such as, stiffness. Results obtained from technical and biological samples are shown illustrating different functions and the versatility of the presented tool. We assert that, this highly precise tractable tool paves the way to a new set of comprehensive studies in medicine, biology and materials science.
\end{abstract}

\section{Background}

The classical diffraction limit for the resolution of an optical microscope can be overcome (Hell 2009) and that was demonstrated in the last years. Super-resolution techniques based on stimulated emission depletion (STED) and others (Schermelleh et al. 2010) showed their potential in material science applications and biological sciences (Willig et al. 2006). Nowadays, most of these techniques are commercially available and are routinely used on live biological samples (Pellett et al. 2011). The most inspiring aspect for all these ultrahigh resolution approaches is the promise of theoretically unlimited resolution (Hell 2003).

The basic idea of STED is to selectively switch off the periphery of the fluorescent focus by superimposing the diffraction limited excitation focus with a second beam, red shifted in wavelength with respect to the excitation wavelength. This second beam stimulates the excited dye molecule to release its energy across an energy transition which corresponds to the same energy, i.e. same wavelength, like the stimulation beam itself. Hence the fluorescence emission is inhibited in those areas where the second beam falls and the dye molecule appears dark to the detector. By adding an appropriate phase mask, the focal distribution of the stimulating beam can be tuned into a doughnut-like shape featuring

\footnotetext{
* Correspondence: benjamin.harke@iit.it; alberto.diaspro@iit.it

${ }^{\dagger}$ Equal contributors

${ }^{1}$ Nanophysics, Istituto Italiano di Tecnologia, via Morego 30, 16163 Genova, Italy

${ }^{3}$ Department of Physics, University of Genoa, 16146 Genoa, Italy

Full list of author information is available at the end of the article
}

zero intensity at its center. Now, exciting the molecules with a regular excitation laser beam and de-exciting them to its ground state with a donut shaped STED laser beam leaves fluorescent molecules in an area of sub-diffraction dimensions. Saturating the stimulated emission depletion process by increasing the power of the STED beam further reduces the size of the fluorescing area and thus attains 'super resolution'. In contrast to any currently used super resolution techniques, STED microscopy possesses a unique set of advantages, such as, rapid image acquisition that enables to study fast dynamics; for example, vesicle movement in vivo, dendritic spine activity or actin dynamics within living brain cultures (Westphal et al. 2008); (Nägerl et al. 2008); (Urban et al. 2011). A further advantage is the ability of being adapted to other fluorescent based techniques as it has been shown during the last decade for fluorescence correlation spectroscopy (FCS, (Eggeling et al. 2008)), fluorescence lifetime image (FLIM (Lenz et al. 2011)), fluorescence recovery after photobleaching (FRAP (Sieber et al. 2007)), etc. Multicolor imaging (Pellett et al. 2011), time resolved and steady state fluorescent probing (Mueller et al. 2011) have been illustrated inside biological membranes. Hence, among the competing high resolution techniques, STED draws own rubric line and prevails as the suitable candidate for high resolution probing for our requisite.

Near field microscopes, such as atomic force microscope (AFM) (Binnig et al. 1986), work label-free and evince an excellent resolution. The underlying principle is to scan the surface with a sharp tip obtaining the

\section{空}

(c) 2012 Harke et al; licensee Springer. This is an Open Access article distributed under the terms of the Creative Commons Attribution License (http://creativecommons.org/licenses/by/2.0), which permits unrestricted use, distribution, and reproduction in any medium, provided the original work is properly cited. 
height information of the investigated sample, thus enabling the reconstruction of the sample topography (Stark et al. 2001). Tip based investigations in aqueous conditions has been of wide interest for the research in biological samples. Today, this is a standard AFM application that can provide high resolution topography on a large class of biomaterials, such as proteins, model membranes and cells (Dante et al. 2011); (Madl et al. 2006). The diversity in growth of AFM implementing fluorescence strategies like FCS, FRAP, FLIM, Förster resonance energy transfer (FRET), etc. (García-Sáez and Schwille 2010); (Micic et al. 2004); (Vickery and Dunn 2001) show the potential to target various queries that came up in routine morphological analysis.

In general, a label-free technique like AFM works non-specifically and shows thereby a drawback for a variety of biological studies. In particular, in the study of complex systems, such as cells, the investigation is often focused on a specific cellular distribution within the whole cell network complex. An optical fluorescence microscope platform can give this specificity and is, therefore, often seen together with the AFM (Kassies et al. 2005). The information gained from the AFM correlated with a color-encoded molecular fluorescence map is distinctly an important tool for deeper interpretation of the results.

We take a step forward into nanoscopic domains by combining these two key technologies AFM and STED microscopy. The AFM covers a vast field of queries and hence this step of coupling with STED is an inevitable one in order to answer sub diffraction level morphology questions supported with fluorescence specificity. It is important to realize that we overcome limitations of individual setups with a combined setup, for example: the performance of AFM is limited by non-specificity and resolution of STED is experimentally limited by available laser power for depletion and photo-stability of the dye used (Harke et al. 2008); (Hotta et al. 2010). Pre-indication of sample distribution can help to configure the AFM correctly and similarly, photo exposure can be avoided in areas, if identified by morphology. Thus, both techniques support each other in a way other techniques would not. This reliability and the precision of measurements reach nano dimensions by avoiding artifacts (Battaglia et al. 2011); (Sharma S 2999), providing an accurate chemical identification of the features by fluorescence.

Force spectroscopy (Domke and Radmacher 1998); (Butt et al. 2005); (Müller and Dufrêne 2011) is another AFM mode that can determine mechanical/structural properties of the sample. This method was fruitful in investigating the mechanical properties of cells and cell compartments by collecting a map of force-distance (FD) curves (see Additional file 1) (Schäpe et al. 2009). By indenting the cellular surface, it is possible to obtain the local stiffness of the cell with a lateral resolution in the order of tens of nanometers restricted by environment, tip and the force applicable (Le Grimellec et al. 1998). In such cases, the knowledge of the tip position within the cell complex is very significant and STED plays an important role here, by doing so. Fluorescence image from a conventional microscope can only make a limited contribution to these force measurement studies since the lateral resolution of the light microscope is about one order of magnitude lower than the one obtained by the AFM. This problem gets graver in densely packed samples like cellular complexes or a heavily aggregating species. With all these points stated above, it is apparent that AFM-STED promises a nanoscopic manipulation scenario with specificity and precision.

\section{Methods}

The setup used for the experiments is based on a conventional multi-photon microscope platform with an oil immersion lens as objective for all optical imaging (NA 1.4, 63X paired on Nikon A1 MP R, Nikon Instruments, Japan). Excitation of the sample was performed by a pulsed laser diode with a wavelength of $640 \mathrm{~nm}$ (Picoquant, Germany) and for the STED depletion beam the light was generated by an femtosecond modelocked Titanium:Sapphire laser (Chameleon Vision II, Coherent, USA) and spatially and temporally shaped as previously described in the literature (Harke 2008) to generate a donut like intensity distribution in the focal area enhancing resolution uniformly along the lateral dimensions. The implementation of the near field method on this setup was realized by mounting an AFM scan head (NanoWizard $^{\circledR}$ II, JPK Instruments AG, Germany) on an isolated sample stage (JPK Instruments AG, Germany) which replaced the original microscope stage. The data was acquired by a separate controller. which talks to the AFM computer through a rapid data bus for real time 3D illustration of the tip scanning measurements. A schematic of the setup arrangement is shown in Figure 1a.

Coarse tip alignment over the sample surface can be done by looking at the laser reflection measurements (Figure 1b) close to the sample substrate. For finer alignment of the tip over a desired location, an optical calibration has to be carried out before-hand in order to avoid possible optical aberrations from optical elements like scan lens and tube lens in the microscope beam scanning path. This calibration follows a standard routine in the AFM software (DirectOverlay ${ }^{\text {TM }}$, JPK Instruments AG, Germany) and has to be done once for any given scan field. Later on, the AFM software will be able to process and merge the optical image to AFM scans at any position, orientation and scan direction of the tip in that scan field (see Additional file 1). A 3D scheme of the above mentioned setup is shown in 


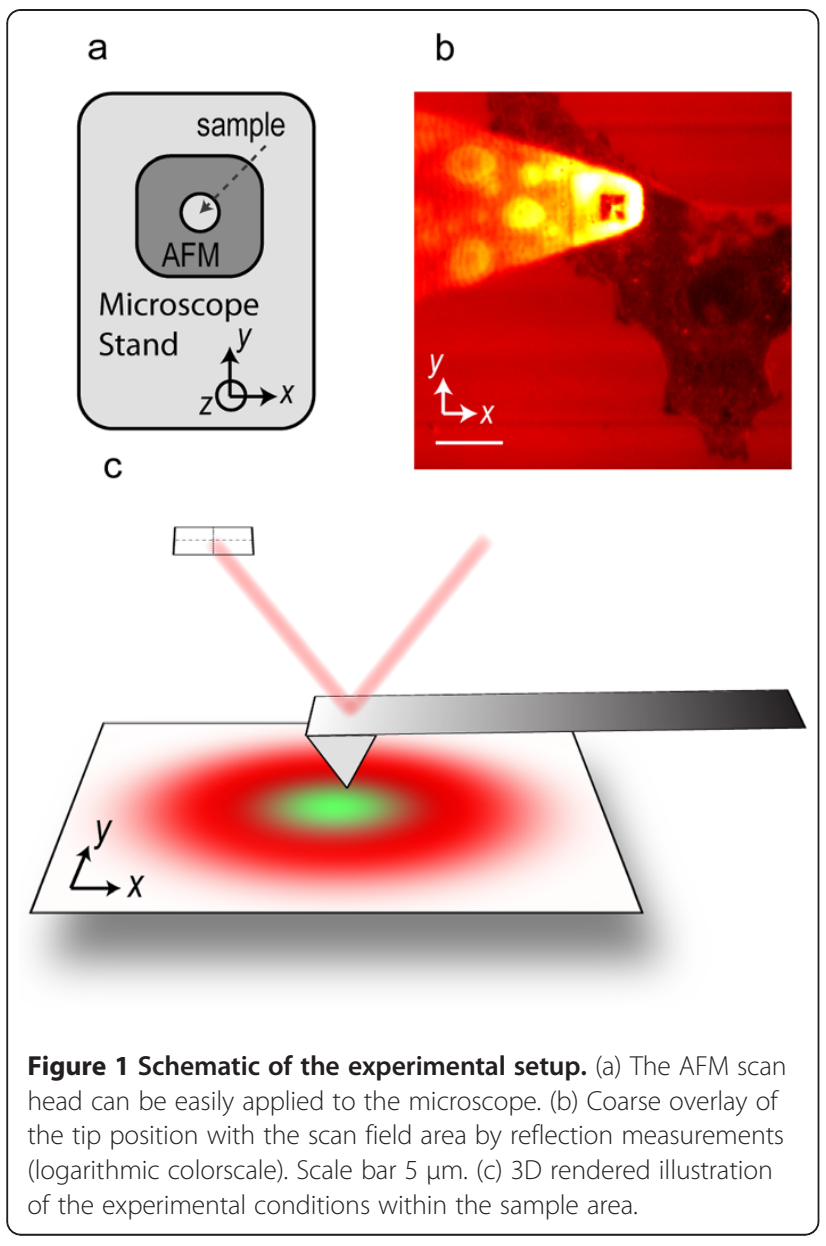

Figure 1c. Both these techniques can run independently of each other and also simultaneously. However, at the time of fine AFM scans, the oil immersion objective lens of the optical microscope was retracted from the sample surface due to the sensitive nature of AFM to environmental noise. A calibration sample was prepared by adhering nominal $40 \mathrm{~nm}$ fluorescing crimson spheres on a glass coverslip (see Additional file 1). This sample was imaged in ambient conditions working in intermittent-contact mode, using single beam silicon cantilevers with a nominal spring constant of $40 \mathrm{~N} / \mathrm{m}$ and a typical tip radius of $10 \mathrm{~nm}$ (TESPA, Nanoworld, Switzerland). Samples with cos7 cells in which microtubuli were immunolabeled by an antibody decorated with ATTO647N (Atto-Tec GmbH, Siegen, Germany) have been prepared by an antibody staining protocol for fixed cells and have been investigated in liquid buffer environment (phosphate buffered saline, PBS). FDcurves have been acquired mounting soft triangular silicone nitride cantilevers with a nominal spring constant of $0.01 \mathrm{~N} / \mathrm{m}$ and a typical tip radius of $20 \mathrm{~nm}$ (MLCT, Bruker, USA)

\section{Results and discussion}

To observe a direct impact of the combinational technique, we imaged fluorescent spheres. Later this type of sample became a routine method for maintenance and performance check due to its simplicity of preparation and stable behavior to environmental perturbations. The setup was tested and the representative measurements are shown in Figure 2. It clearly shows the advantages of the STED routine coupled to an AFM in comparison to the images acquired by the confocal counterpart. In the confocal images, only the rough shape of areas with a higher bead concentration is visible while, in comparison, STED images significantly gain information content in the image (c). AFM imaging of the same area of interest (b) shows the topographical landscape which matches the STED image information: every single $40 \mathrm{~nm}$ fluorescent sphere as well as agglomerated structures can be identified in STED and AFM while the confocal mode fails to do so. For better illustration, each of the images shows an enlargement of an area marked by the dashed square. Within this area, a finer scan was performed by the AFM and the overlay of AFM image with the corresponding confocal image and STED image is shown in $\mathrm{d}$ and e, respectively. To emphasize this fact, a 3D rendered view of the AFM image is presented in combination with the confocal data (f) and the STED data (g). Note that the optical image presented here is raw data without the need of any computational optimization.

The results presented so far are not only proof of principle but also of scientific interest: they point out the benefits these techniques gain when working in together. We take these investigations further by studying a biological cell sample in aqueous conditions which require special attention for the AFM scanning conditions. The measurements are presented in Figure 3a which shows a confocal image of the microtubules of cos7 cells decorated with the label ATTO $647 \mathrm{~N}$ (Atto-Tec GmbH, Germany) in PBS (1x) solution. The STED image of the same area (b) shows the finer features which are directly observable, examining the raw data of the STED image. The acquisition time of this super resolution fluorescence map is orders of magnitude shorter than in AFM mode. With the help of this map we can target a region of interest for further investigation by AFM which significantly reduces the overall sample investigation time and possible tip contamination due to imprecise targeting.

Microtubules are an important component of the cytoskeleton and provide a mechanical support to the cell. Maps of FD curves were acquired. This AFM mode allowed for the determination of the local variation of cell stiffness. In this mode of operation, the tip approaches the sample surface until a certain force has been detected before retracting again. During this process, the deflection of 


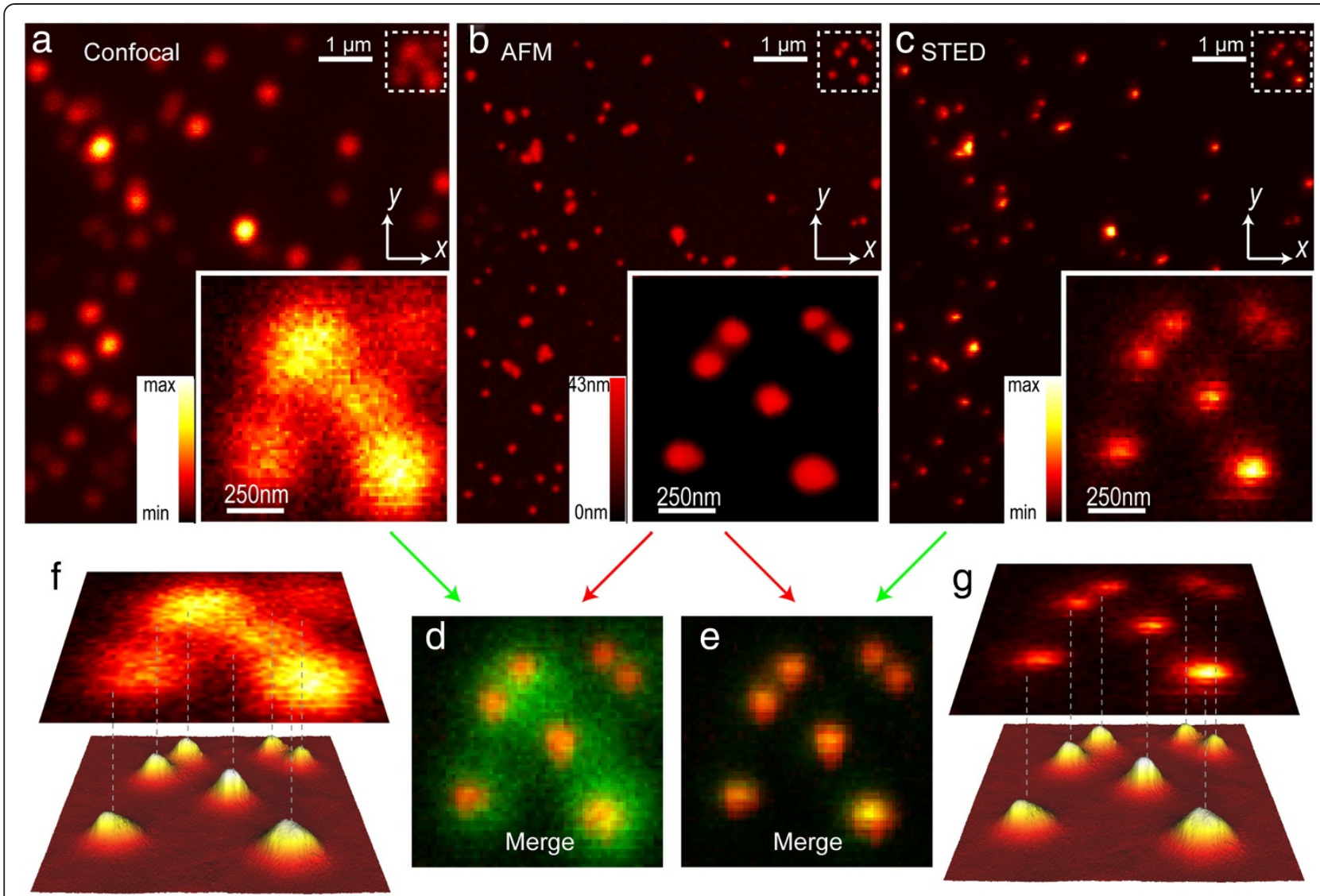

Figure 2 40nm fluorescent spheres super-resolved by STED and spatially overlaid with the corresponding AFM image. Confocal image (a), AFM image (b) and STED image (c) are presented as raw data. For the optical images, the highlighted area is a zoom into the same dataset, in case of AFM a new high pixilated scan was done. Overlay of the AFM image with the confocal scan (d) and with the STED data (e). A 3D rendered view is shown for the confocal mode and the STED mode in ( $f$ and $(g)$, respectively. Optical images are presented as raw data.

the cantilever is recorded to obtain FD curves. One of the advantages of force mapping is the reduction of lateral stress between tip and sample, and the prevention of possible stretching of the sample. Topographical information of the sample can be extracted from the data by a fitting routine (see Additional file 1) and is mapped in 3c. A force spectroscopy map within the area marked in the fluorescence images $3 \mathrm{a}, 3 \mathrm{~b}$ was acquired.

If we examine the images, we see a meshwork with a junction of a tubule bundle and two single filaments. The overlay of the height information with the confocal and STED data is presented in a 3D stack in the Figure 3c, 3d and $3 \mathrm{e}$. From these three images, the confocal data is not able to confirm the AFM data while the STED image exhibits every single filament within the meshwork. The single force curves can now be investigated further in order to extract the Young's modulus which gives information about elasticity of the sample. Since part of the cell cytoskeleton is investigated here, the Young's moduli map should feature the corresponding stiffer parts of the cell. This additional study of the force curves bearing details of the cell elasticity is overlaid with all before-mentioned imaging modes in Figure 3c-3f. The maps for the Young's moduli (f), the STED (e) and confocal data (d) are displayed in perspective according to the $3 \mathrm{D}$ rendered height information (c). Evidently, the Young's moduli map matches with the data extracted from the height analysis and the STED image. The number of the microtubles in the bundle extracted from the STED image can be estimated as three to five since they are clearly distinguished from the rest of the cell area. The two single filaments branching off that bundle, optically visible only with STED, are also traceable in the Young's moduli map of limited contrast by the membrane stiffness variations over the cytoskelton. This unleashes a new set of interpretations which can be supported with STED images like the individual thickness of a fiber, etc.

\section{Conclusion}

In summary, we reported for the first time the combination of AFM imaging with the super resolution STED microscopy. A nominal multi photon fluorescence 

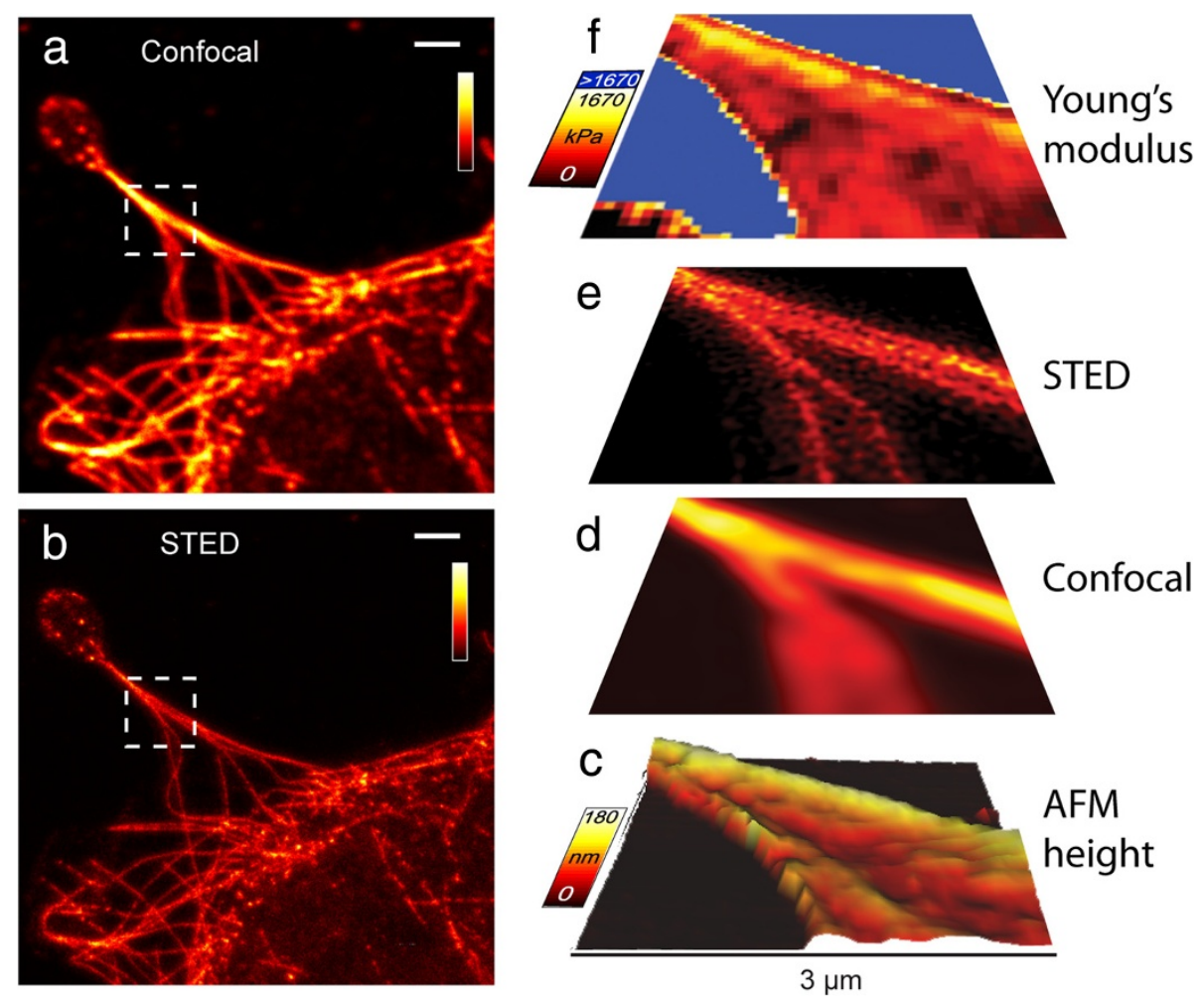

Figure 3 Cos7 cells labeled with Atto $647 \mathrm{~N}$, (a) Confocal image, (b) STED image (both raw data), (c) 3D rendered view of AFM measured height extracted from AFM force curves ( $45 \times 45$ pixilated scan), (d) Confocal image and (e) STED image (both linear deconvolved) (f) Elasticity map calculated from AFM force curves. Scale bars in in (a) and (b): $2 \mu \mathrm{m}$, axes bars in (c)-(f): $3 \mu \mathrm{m}$.

microscope is equipped with a STED performance numbered well below $65 \mathrm{~nm}$ in resolution and boosted by an AFM that facilitates nano probing. This attempt realized on a commercial unit makes the device very attractive for large set of applications by their prompt availability. The optical overlay with the AFM image was first demonstrated on a technical sample under dry conditions and later on a biological sample in fluid environment. Since the structural results achieved by STED imaging match with the AFM recordings, many possible artifacts in each of the techniques can be excluded; moreover structures of interest can be selected and brought into the scientific focus. The work within aqueous conditions in a complex biological cell network illustrates and proves the compatibility to lead to results in diverse biological samples. In the future, the combination of the high resolution STED technique with the different AFM modes can be applied to various kinds of samples and applications where specificity and targeting can enhance the versatility of the AFM. For example, in local adhesion measurements based on a functionalized tip the precise knowledge of the tip position on a living cell is of vital importance in order to correlate an adhesion process to a specific target. In this scenario, the higher precision given by STED microscopy also reduces the total number of force spectroscopy cycles for the adhesion determination which thereby reduces the risk of possible tip contamination. In addition, the overall sample investigation time will be significantly shortened which is essential, in particular, in living tissue.

In the decade of nano manipulation inside cells, AFM STED probing is one of the most prominent candidates.

\section{Additional file}

Additional file 1: Supporting Information

Abbreviations

STED: Stimulated emission depletion; AFM: Atomic Force Microscopy; FCS: Fluorescence Correlation Spectroscopy; FLIM: Fluorescence Lifetime Imaging; FRET: Forsters Resonance Energy Transfer; FRAP: Fluorescence Recovery After Photobleaching; NA: Numerical Aperture; MP: Multiphoton; FD: Force Distance; PBS: Phosphate buffer saline.

\section{Competing interests}

The authors declare that they have no competing interests. 


\section{Acknowledgements}

We wish to thank Dr. Andrea Raimondi, Neuroscience and Brain technologies, ITT, Genoa for preparation and staining of the cells needed for the experiments. The authors also wish to thank Nikon Instruments S.p.A as well as JPK Instruments, in particular Torsten Jähnke, for the technical collaboration.

\section{Author details}

'Nanophysics, Istituto Italiano di Tecnologia, via Morego 30, 16163 Genova, Italy. ${ }^{2}$ JPK Instruments AG, Bouchéstrasse 12, 12435 Berlin, Germany. ${ }^{3}$ Department of Physics, University of Genoa, 16146 Genoa, Italy.

\section{Authors' contributions}

$\mathrm{AD}$ and $\mathrm{BH}$ conceived and supervised the project. BH drafted the manuscript and made the optical design of the setup. JVC carried out the measurements and the alignment and worked on the draft. $\mathrm{HH}$ helped setting up the AFM and refined the manuscript. CC helped performing the AFM measurements and analyzing the data and refined the manuscript. AD has given final approval of the manuscript.

Received: 27 January 2012 Accepted: 25 April 2012

Published: 25 April 2012

\section{References}

Battaglia G, LoPresti C, Massignani M et al (2011) Wet nanoscale imaging and testing of polymersomes. Small 7:2010-2015. doi:10.1002/smll.201100511

Binnig G, Quate CF, Gerber C (1986) Atomic force microscope. Phys Rev Lett 56:930-933

Butt HJ, Cappella B, Kappl M (2005) Force measurements with the atomic force microscope: Technique, interpretation and applications. Surf Sci Rep 59:1-152

Dante S, Haufl T, Steitz R et al (2011) Nanoscale structural and mechanical effects of beta-amyloid (1-42) on polymer cushioned membranes: A combined study by neutron reflectometry and AFM Force Spectroscopy. Biochimica et Biophysica Acta (BBA)-Biomembranes 1808:2646-2655

Domke J, Radmacher M (1998) Measuring the elastic properties of thin polymer films with the atomic force microscope. Langmuir 14:3320-3325

Eggeling C, Ringemann C, Medda R et al (2008) Direct observation of the nanoscale dynamics of membrane lipids in a living cell. Nature 457:1159-1162

García-Sáez AJ, Schwille P (2010) Surface analysis of membrane dynamics. Biochimica et Biophysica Acta (BBA) - Biomembranes 1798:766-776. doi:10.1016/j.bbamem.2009.09.016

Harke B (2008) 3D STED microscopy with pulsed and continuous wave lasers. Niederslächsische Staats-und Universitlätsbibliothek Glöttingen

Harke B, Keller J, Ullal CK et al (2008) Resolution scaling in STED microscopy. Opt Express 16:4154-4162

Hell SW (2003) Toward fluorescence nanoscopy. Nat Biotechnol 21:1347-1355

Hell SW (2009) Microscopy and its focal switch. Nat Methods 6:24-32. doi:10.1038/nmeth.1291

Hotta J, Fron E, Dedecker P et al (2010) Spectroscopic rationale for efficient stimulated-emission depletion microscopy fluorophores. J Am Chem Soc 132:5021-5023

Kassies R, Van Der Werf KO, Lenferink A et al (2005) Combined AFM and confocal fluorescence microscope for applications in bio-nanotechnology. J Microsc 217:109-116. doi:10.1111/j.0022-2720.2005.01428.x

Le Grimellec C, Lesniewska E, Giocondi MC et al (1998) Imaging of the surface of living cells by low-force contact-mode atomic force microscopy. Biophys $J$ 75:695-703

Lenz MO, Brown ACN, Auksorius E, et al. (2011) A STED-FLIM microscope applied to imaging the natural killer cell immune synapse. p 79032D-79032D-6

Madl J, Rhode S, Stangl H et al (2006) A combined optical and atomic force microscope for live cell investigations. Ultramicroscopy 106:645-651

Micic M, Hu D, Suh YD et al (2004) Correlated atomic force microscopy and fluorescence lifetime imaging of live bacterial cells. Colloids Surf B Biointerfaces 34:205-212. doi:10.1016/j.colsurfb.2003.10.020

Mueller V, Ringemann C, Honigmann A et al (2011) STED Nanoscopy Reveals Molecular Details of Cholesterol-and Cytoskeleton-Modulated Lipid Interactions in Living Cells. Biophys J 101:1651-1660

Müller DJ, Dufrêne YF (2011) Force nanoscopy of living cells. Curr Biol 21:R212R216. doi:10.1016/j.cub.2011.01.046

Nägerl UV, Willig Kl, Hein B et al (2008) Live-cell imaging of dendritic spines by STED microscopy. Proc Natl Acad Sci 105:18982
Pellett PA, Sun X, Gould TJ et al (2011) Two-color STED microscopy in living cells. Biomed Opt Express 2:2364-2371. doi:10.1364/BOE.2.002364

Schäpe J, Prausse S, Radmacher M, Stick R (2009) Influence of lamin A on the mechanical properties of amphibian oocyte nuclei measured by atomic force microscopy. Biophys J 96:4319-4325

Schermelleh L, Heintzmann R, Leonhardt H (2010) A guide to super-resolution fluorescence microscopy. J Cell Biol 190:165-175

Sharma S, Santiskulvong C, Bentolila LA, et al. Correlative nanomechanical profiling with super-resolution F-actin imaging reveals novel insights into mechanisms of cisplatin resistance in ovarian cancer cells. Nanomedicine: Nanotechnology, Biology and Medicine. doi:10.1016/j.nano.2011.09.015

Sieber JJ, Willig Kl, Kutzner C et al (2007) Anatomy and dynamics of a supramolecular membrane protein cluster. Science 317:1072

Stark M, Möller C, Müller DJ, Guckenberger R (2001) From images to interactions: high-resolution phase imaging in tapping-mode atomic force microscopy. Biophys J 80:3009-3018

Urban NT, Willig KI, Hell SW, Nägerl UV (2011) STED nanoscopy of actin dynamics in synapses deep inside living brain slices. Biophys J 101:1277-1284

Vickery SA, Dunn RC (2001) Combining AFM and FRET for high resolution fluorescence microscopy. J Microsc 202:408-412. doi:10.1046/j.1365-2818.2001.00857x

Westphal V, Rizzoli SO, Lauterbach MA et al (2008) Video-rate far-field optical nanoscopy dissects synaptic vesicle movement. Science 320:246

Willig Kl, Rizzoli SO, Westphal V et al (2006) STED microscopy reveals that synaptotagmin remains clustered after synaptic vesicle exocytosis. Nature 440:935-939

doi:10.1186/2192-2853-1-3

Cite this article as: Harke et al: A novel nanoscopic tool by combining AFM with STED microscopy. Optical Nanoscopy 2012 1:3.

\section{Submit your manuscript to a SpringerOpen ${ }^{\mathcal{D}}$ journal and benefit from:}

- Convenient online submission

- Rigorous peer review

- Immediate publication on acceptance

- Open access: articles freely available online

- High visibility within the field

- Retaining the copyright to your article

Submit your next manuscript at $>$ springeropen.com 